\title{
Silencing LPAAT $\beta$ inhibits tumor growth of cisplatin-resistant human osteosarcoma in vivo and in vitro
}

\author{
LEI SONG ${ }^{1}$, PING DUAN ${ }^{2}$, YIBO GAN $^{1}$, PEI LI $^{1}$, CHEN ZHAO $^{1}$, \\ JIANZHONG XU ${ }^{1}$, ZEHUA ZHANG ${ }^{1}$ and QIANG ZHOU ${ }^{1}$ \\ ${ }^{1}$ Department of Orthopaedics and ${ }^{2}$ Southwest Eye Hospital, First Affliated Hospital, \\ Third Military Medical University, Chongqing 400038, P.R. China
}

Received October 11, 2016; Accepted December 6, 2016

DOI: $10.3892 /$ ijo.2016.3820

\begin{abstract}
Cisplatin-resistance has become a major impediment in the medical treatment of cancers such as osteosarcoma, the most common primary malignancy of bone. Since lysophosphatidic acid acyltransferase $\beta$ (LPAAT $\beta$ ) was reported to be critically involved in osteosarcoma, our study investigated the role of LPAAT $\beta$ in human osteosarcoma with cisplatinresistance. Expression of LPAAT $\beta$ or other relevant proteins were analyzed in 40 osteosarcoma patients by immunohistochemistry analysis (IHC), and in cisplatin-resistant sublines by real-time PCR and western blotting. Next, the synthesized siRNA was inserted into the lentivirus vector and silencing of LPAAT $\beta$ expression was employed to determine the effect of LPAAT $\beta$ on cisplatin-resistant osteosarcoma cell viability in vitro and osteosarcoma tumor growth in vivo with cisplatin treatment. Exogenous LPAAT $\beta$ mediated by heritable RNAi decreased cisplatin-resistant sensitivity through activating the PI3K/Akt/mTOR signaling pathway. We further demonstrate that silencing LPAAT $\beta$ effectively inhibited tumor growth in nude mice with xenografts of cisplatin-resistant osteosarcoma cells. IHC assay results showed that PI3K/Akt/mTOR signaling pathway was also involved in this process. Our results suggested that LPAAT $\beta$ may play an important role in osteosarcoma and silencing LPAAT $\beta$ may be exploited as a novel therapeutic strategy for the clinical management of cisplatin-resistance.
\end{abstract}

\section{Introduction}

Osteosarcoma is the most common primary malignant tumor that arises from osteoid tissues in young adults and adoles-

Correspondence to: Professor Qiang Zhou, Department of Orthopaedics, First Affliated Hospital, Third Military Medical University, 30 GaoTanYan, Chongqing 400038, P.R. China E-mail: qiangzhou2016@163.com

Key words: osteosarcoma, LPAAT $\beta$, RNAi, cisplatin-resistance, signaling pathway cents $(1,2)$. Most patients with osteosarcoma are diagnosed at a late stage with strong requirement of surgical radiotherapy or adjuvant chemotherapy, however, usually it is not effective $(3,4)$. Since platinum-based drugs such as cisplatin are the standard first-line agents used alone or in combination with other drugs for osteosarcoma treatment, osteosarcoma cells may acquire resistance to cisplatin with tumor recurrence through the expansion of cisplatin-resistance cell population $(5,6)$. Unfortunately, a major challenge exists in bone cancer fields due to the limited efficiency of current therapeutic strategies. Therefore, there is an urgent need to develop novel early molecular markers of therapeutic targets for osteosarcoma with cisplatin-resistant scenario $(7,8)$.

Lysophosphatidic acid acyltransferase $\beta$ (LPAAT $\beta$, 1-acylglycerol-3-phosphate O-acyltransferase 2, Agpat2), one of transmembrane proteins, are involved in regulating osteosarcoma cell proliferation $(9,10)$. During this process, LPAAT $\beta$ could activate the acyltransferase activity and convert lysophosphotidic acid (LPA) into phosphatidic acid (PA), such as the mTOR and Raf-1 signaling pathways, which is considered as an important secondary messenger for cell survival and proliferation (11-13). However, the comprehensive underlying molecular mechanisms responsible for LPAAT $\beta$ as a cell viability regulator in osteosarcoma disease remain unclear. Recently LPAAT $\beta$ has become a pharmacological research target of osteosarcoma. It was reported that overexpression of miR-24 inhibited osteosarcoma cell proliferation both in vitro and in vivo through binding to 3 'UTR of LPAAT $\beta$ (14). Other research revealed that exogenous expression of LPAAT $\beta$ effectively promoted osteosarcoma tumor growth, while knockdown of which inhibited tumor growth in vitro (15). However, the effect of LPAAT $\beta$ expression alteration on human osteosarcoma with cisplatin-resistant environment is not well known.

We examined the LPAAT $\beta$ expression in tumor samples from 40 osteosarcoma patients and then analyzed the relationship between LPAAT $\beta$ expression and clinical behavior of osteosarcoma. We further generated cisplatinresistant sublines using lentivirus-mediated RNAi and fianlly mechanistically investigated the functional role of LPAAT $\beta$ and PI3K/Akt/mTOR signaling pathway involve- 
ment in cisplatin-resistant environment of osteosarcoma in vivo and in vitro.

\section{Materials and methods}

Clinical specimens and samples collection. Cancer tissue specimens were obtained from 40 osteosarcoma patients aged from 13 to 46 years who had undergone resection at the Orthopaedics Department of First Affliated Hospital, Third Military Medical University between 2014 and 2016. Clinical information of all the patients was collected and shown in Table I. This study was approved by the ethics committee of First Affliated Hospital, Third Military Medical University, and all patients provided informed consent.

Ethical approval. All procedures performed in studies involving human participants were in accordance with the ethical standards of the institutional and/or national research committee and with the 1964 Helsinki Declaration and its later amendments or comparable ethical standards. All animal experiments were carried out strictly in accordance with international ethical guidelines and the National Institutes of Health Guide concerning the Care and Use of Laboratory Animals. The experiments were approved by Medical Ethics Committee of the Southwest Hospital and the First Affiliated Hospital of the Third Military Medical University.

Cell culture. Two human osteosarcoma cell lines MG-63 and SaOS-2 [American Type Culture Collection (ATCC), Manassas, VA, USA] were maintained in DMEM high glucose media (PAA Laboratories, Pasching, Austria) supplemented with $10 \%(\mathrm{v} / \mathrm{v})$ fetal bovine serum (PAA Laboratories, Pasching, Austria) at $37^{\circ} \mathrm{C}$ and $5 \% \mathrm{CO}_{2}$. Culture medium was changed twice a week, and splitting of the cell culture was done every ten days at confluency of 70-80\%. Cells were kept in a $5 \% \mathrm{CO}_{2}$ atmosphere at $37^{\circ} \mathrm{C}$ before analyzing.

Acute cytotoxicity assay. The acute cytotoxic effects of cisplatin $(1.5,3,4.5,6,8$ and $4 \mu \mathrm{M}, 8,12$ and $16 \mu \mathrm{M})$ on cell viability were measured in confluent monolayers in 96-well plates, using the CCK8 kit (Sigma-Aldrich) according to the standard method. Briefly, cells were allowed to grow in a $75-\mathrm{cm}^{2}$ cell culture flask (TPP Techno Plastic Products, Trasadingen, Switzerland) until $95 \%$ confluent. The cells were then seeded into each well and incubated for $24 \mathrm{~h}$ at $37^{\circ} \mathrm{C}$ in an atmosphere of $7.5 \% \mathrm{CO}_{2}$ in air. In the last hour of incubation, $100 \mu \mathrm{lCCK} 8$ solution (Dojindo, Japan) was added to each well for $1 \mathrm{~h}$, and the absorbance was read at $450 \mathrm{~nm}$ on the BioTek FL600 ${ }^{\circledR}$ fluorescence/absorbance plate reader (BioTek Instruments Inc., Winooski, VT, USA). Control groups consisted of cells in media (minus chemical) which were processed identically and incubated simultaneously as treated groups. Parallel sets of wells without cells were incubated as process blanks. Calculation of inhibitory concentration $50\left(\mathrm{IC}_{50}\right)$ value was determined from the absorbance versus concentration curve for each chemical based on a minimum of 3 experiments performed at separate times.

Establishment of cisplatin-resistant subline clonogenic assay. A monoclonal strain was separated by dilution culture method.
MG-63 and SaOS-2 were derived by incubation with stepwise increasing cisplatin concentrations (from 1.5 to $16 \mu \mathrm{M}$ ). Cells were seeded in a 24-well dish at 100 cells/well and allowed to adhere for $24 \mathrm{~h}$ in an incubator after which the cisplatin (Sigma, USA) was added. Cells were replaced with fresh DMEM media after 24-h incubation. After each treatment, the survival cells re-expanded and were conventionally propagated for four generations in cisplatin-free media. Each individual experiment was performed in triplicate and repeated three times.

Quantitative real-time PCR. Total RNA ( $2 \mu \mathrm{g})$ was extracted using an RNeasy Mini kit (Qiagen, Germany). RNA purity and concentration were estimated with an ND-1000 spectrophotometer (NanoDrop Technologies, Thermo Scientific, UK). Total messenger RNA was reverse-transcribed into cDNA using the Verso ${ }^{\mathrm{TM}}$ cDNA synthesis kit (Thermo Fisher Scientific) as described by the manufacturer. Each forward and reverse validated primer $(5 \mu \mathrm{M})$ (Sigma-Aldrich, UK) is listed in Table II. Each reaction contained $50 \mathrm{ng}$ cDNA and was carried out using SYBR ${ }^{\circledR}$ green RT-PCR master mix (Life Technologies). PCR reactions with $50 \mathrm{ng} / \mu 1$ of the cDNA samples per $10 \mu \mathrm{l}$ final reaction volume, were performed using standard cycling parameters (stage $1,50^{\circ} \mathrm{C}$ for $2 \mathrm{~min}$, stage 2, $95^{\circ} \mathrm{C}$ for $10 \mathrm{~min}$ then 40 cycles of $95^{\circ} \mathrm{C}$ for $15 \mathrm{sec}$ and $60^{\circ} \mathrm{C}$ for $1 \mathrm{~min}$ ) on an ABI $7900 \mathrm{HT}$ sequence detection system. Normalization was performed using GAPDH as the internal control, and relative gene expression was calculated by the comparative $2^{-\Delta \Delta \mathrm{Ct}}$ method using SDS 2.2 software (Applied Biosystems).

Western blot analysis. Before employing a set of phosphospecific antibodies, lysis buffer (12.5 ml Tris-HCl, 2 g SDS, $10 \mathrm{ml}$ glycerol, $67.5 \mathrm{ml}$ distilled water) was used to harvest whole-cell lysates, followed by sonication. The concentration of protein in the cell lysates was estimated by using a bicinchoninic acid (BCA) assay. Novex ${ }^{\circledR} 4-20 \%$ Tris-glycine 12-well polyacrylamide gradient gels (Invitrogen, UK) were used to separate proteins. Subsequently, proteins were transferred onto a nitrocellulose membrane (GE Healthcare, Little Chalfont, UK) via the Protean Mini Cell system (Bio-Rad, München, Germany). After blocking in 5\% non-fat milk in TBS/0.1\% Tween-20 (Merck, Darmstadt, Germany) (2 h, RT), the membrane was incubated with the corresponding primary antibody (overnight, $4^{\circ} \mathrm{C}$ ). After washing with TBS $/ 0.1 \%$ Tween-20 the secondary (peroxidase-conjugated) antibody was added (1:10,000, $2 \mathrm{~h}, \mathrm{RT})$. For visualization of the bound antibodies the Fusion FX7 imaging system (PeqLab, Erlangen, Germany) was used. All antibodies were diluted in 5\% milk/1X TBS-Tween (w/v). Enhanced chemiluminescence (GE Life Sciences) and X-ray film (Fujifilm) were finally used to visualize the proteins. Sections were incubated with the primary antibody, anti-human LPAAT $\beta$ polyclonal antibody (1:10,000; Santa Cruz), anti-human P-gp polyclonal antibody (1:10,000; Abcam), anti-human MRP1 polyclonal antibody (1:4,000; Abcam), anti-human GST polyclonal antibody (1:4,000; Abcam), anti-human Bcl-2 polyclonal antibody (1:4,000; Santa Cruz); anti-human Akt1/2 polyclonal antibody (1:4,000; Santa Cruz), anti-human Akt1/2 polyclonal antibody (1:4,000; Santa Cruz), anti-human mTOR polyclonal antibody (1:5,000; Santa Cruz) and anti-human PIK3CA polyclonal 
Table I. Clinicopathological features of osteosarcoma in all study subjects.

\begin{tabular}{lc}
\hline Clinicopathological features & No. of cases $(\mathrm{n}=40)$ \\
\hline Age (years) & 24 \\
$\leq 25$ & 16 \\
$>25$ & \\
Gender & 29 \\
Male & 11 \\
Female & \\
Tumor site & 18 \\
Femur & 14 \\
Tibia & 7 \\
Humeral bone & 1 \\
Other & \\
Pathological fracture & 15 \\
Absent & 25 \\
Present & \\
Recurrence & 27 \\
Absent & 13 \\
Present & \\
Response to preoperative & \\
chemotherapy & \\
Good & \\
Poor & \\
N/A & \\
\hline & \\
&
\end{tabular}

antibody (1:10,000; Santa Cruz), respectively, at $4^{\circ} \mathrm{C}$ overnight.

Immunohistochemistry analysis. We detected the expression of LPAAT $\beta$ in tissue samples and phospho-mTOR in tumor samples by immunohistochemisty in nude mice. Samples were carefully digested and fixed overnight in $4 \%$ paraformaldehyde with a $0.1 \mathrm{M}$ phosphate buffer solution ( $\mathrm{pH}$ 7.4). Then cells were embedded in paraffin and sliced into 5 - $\mu \mathrm{m}$-thick serial sections using a Microtome (Leica Microsystems Inc., Buffalo Grove, IL, USA). The sections were deparaffinized and fixed after hydration. Sections were incubated with the primary antibody, anti-human LPAAT $\beta$ polyclonal antibody (1:100; Abcam), anti-mouse phospho-mTOR polyclonal antibody (1:50; Abcam), respectively. at $4^{\circ} \mathrm{C}$ overnight. The photographs of the identified adjacent areas were taken under high magnification $(x 400)$.

Lentivirus-mediated gene silencing. To further prove the importance of LPAAT $\beta$ in the process of cisplatin resistance, small intering RNA (siRNA GCCGGACGGUGGAGAACAUGA) transfection was performed using three sequences designed to target LPAAT $\beta$ which were designed by the Invitrogen RNAi design tool (http://www.invitrogen.com) and synthesized by Invitrogen, Ltd. Non-targeting negative control of siRNA (control TTCTCCGAACGTGTCACGTTTC) was also
Table II. Sequences of the gene-specific primers used for real-time PCR detection in vitro.

\begin{tabular}{ll}
\hline Gene & \multicolumn{1}{c}{ Primer sequence (5'-3') } \\
\hline LPAAT $\beta$ & F: CGGACCGATGTTCGCCGCCATGAACGG \\
& R: AATCTTCCATCATCGCACTTGTAGTTGC \\
P-gp & F: AGTTCGTAGGGCTAGCTAATCGATCGAT \\
& R:CGCACGTGATCGATCCGTCCCGATCGAT \\
MRP1 & F: AGGCCCCTAGCTAGTAGCTAGCCATCG \\
& R: GTAGCTAAAACGTAGCTGTAGCCCTA \\
GST & F: ATAGATATCAGTCCCCCTAGTAGCTAG \\
& R: CTCGAAAATCGATCGTAGTCGATGCC \\
bcl-2 & F: CGCGCTAGCTAGACGCGCGATGATCC \\
& R: CGCGCTAGCTAGTAAGCTAGCTCTAGC \\
PIK3CA & F: CGGGATCGATCCCCGATCGTATAGCT \\
& R: AGATCGATCGTATATCGATAGCTAAC \\
Akt1 & F: CGCGATCTATAGCTCGCCTAGCTAGC \\
& R: GTAGTCTAGCGCGCCCCTAGCTAGAT \\
Akt2 & F: CGCGATGATCGTAGTAGCTAGCTAAC \\
& R: GCTAGATAGCGCTAGCTGACCGATCGC \\
FTOR & F: CGCATACGTAGCTAGCTAGCAACGAC \\
& R: GTAGCTAGCCCTAGCTAGACTGCGCG \\
& F: CGGAGTCAACGGATTTGGTCGTAT \\
& R: AGCCTTCTCCATGGTGGTGAAGAC \\
&
\end{tabular}

F, forward; R, reverse.

synthesized. The oligonucleotides were annealed and inserted into the pLKO.1 siRNA expression vector. The LPAAT $\beta$ interference cell lines MG-63 and SaOS-2 were established using lentivirus transfection and puromycin selection with the second sequence. For lentivirus-mediated RNAi, the 293T cells were selected for transfection with two kinds of auxiliary packaging vector plasmids (LPAAT $\beta$-siRNA-pLKO.1 and pLKO.1) respectively with polyethylenimine (PEI). The supernatant was collected, and MG-63, MG-63-CR, SaOS-2 and $\mathrm{SaOS}-2-\mathrm{CR}$ cells were infected for $5 \mathrm{~h}$ with polybrene. To obtain the stable cell line, puromycin selection was performed. The efficiency of LPAAT $\beta$ inhibition was evaluated by RT-PCR.

Immunofluorescence staining. Cells were plated onto coverslips in MEM medium with $10 \%$ FBS for $24 \mathrm{~h}$ before being transfected with lentivirus-mediated siRNA or negative control. At $48 \mathrm{~h}$ after transfection, the cells were fixed with $4 \%$ paraformaldehyde for $20 \mathrm{~min}$, incubated in $0.3 \%$ Triton $\mathrm{X}-100$-PBS for $10 \mathrm{~min}$ at room temperature, followed by blocking with $5 \%$ goat serum at $37^{\circ} \mathrm{C}$ for $30 \mathrm{~min}$. The cells were then incubated with the primary antibody at $4^{\circ} \mathrm{C}$ overnight; mouse anti-phospho-mTOR IgG (1:200, Santa Cruz). The samples were soaked in goat anti-human IgG conjugated to Cy3 (1:400; Jackson ImmunoResearch) at $37^{\circ} \mathrm{C}$ for $1 \mathrm{~h}$. Subsequently, the nuclei were counter stained 

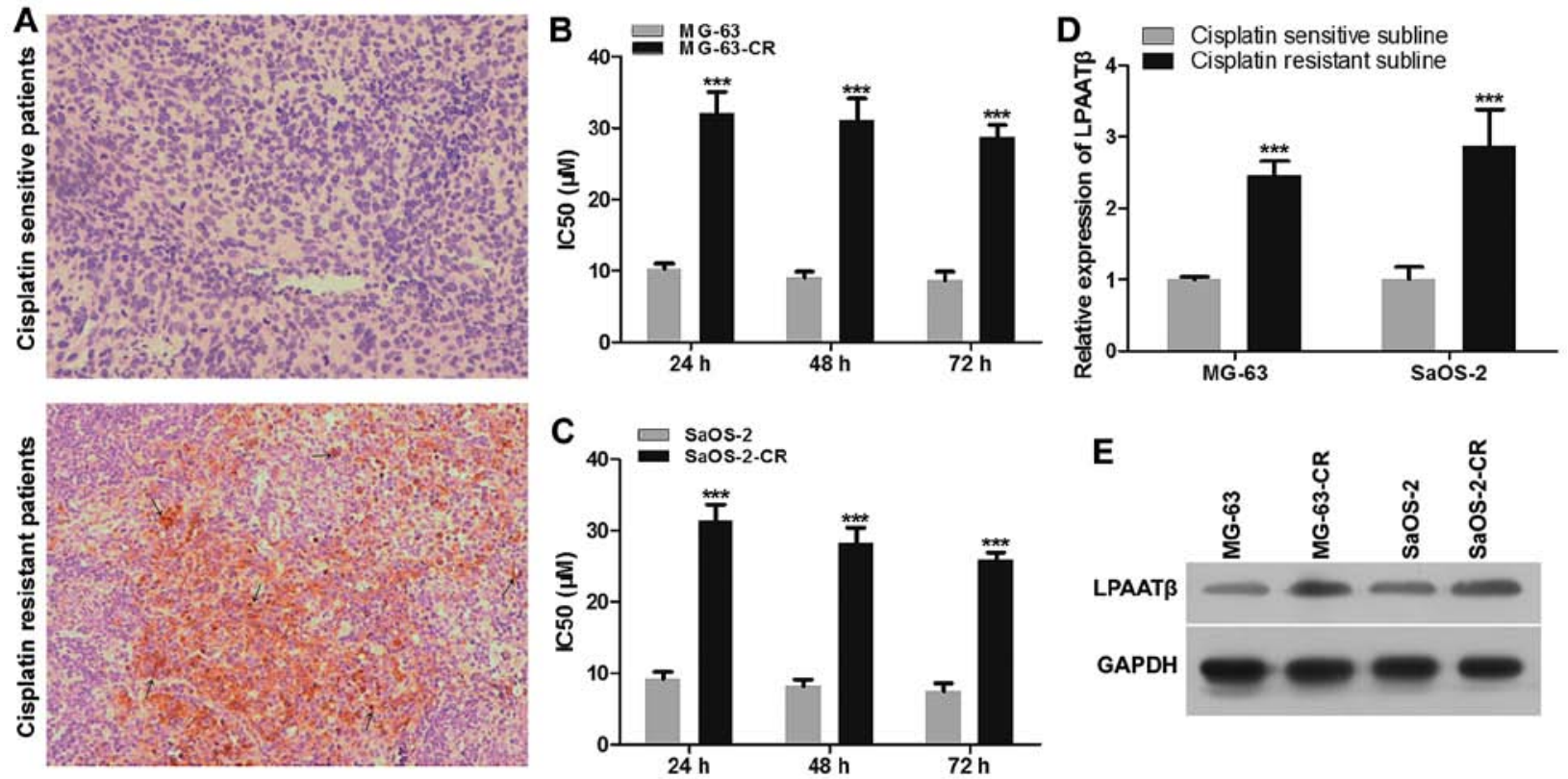

Figure 1. LPAAT $\beta$ level was affected by cisplatin in vivo and in vitro as detected by immunohistochemistry, CCK8 assay, real-time PCR and western blotting. (A) Patients were divided into two cohorts. Cohort I, the osteosarcoma patients with cisplatin-resistant scenario; cohort II, the osteosarcoma patients without cisplatin treatment as the control group. LPAAT $\beta$ expression and distribution in tumor tissue samples collected to perform after surgery, was positive in the cohort I compared to cohort II. The nucleus and membrane of small cells and mature tumor cells are stained brown with LPAAT $\beta$ (indicated by black arrow). (B and C) $\mathrm{IC}_{50}$ of cisplatin in drug-resistant MG-63-CR and SaOS-2-CR cells after cisplatin treatment for 24, 48 and $72 \mathrm{~h}$, compared to parental cells. (D) The mRNA level of LPAAT $\beta$ in drug-resistant MG-63-CR and SaOS-2-CR cells as detected by real-time PCR. (E) Protein level of LPAAT $\beta$ in drug-resistant MG-63-CR and SaOS-2-CR cells as detected by western blotting. ${ }^{* * *} \mathrm{P}<0.001$ vs. negative control ( $\mathrm{n}=3$ experiments).

with DAPI (1:1,000; Sigma-Aldrich, Inc., MO, USA). Images were obtained using an inverted fluorescence microscope (Olympus). The primary antibody was replaced with PBS as the negative control.

Animal experiments. Divided into 4 groups ( $\mathrm{n}=32)$, right flank of SCID mice were inoculated subcutaneously with cisplatin-resistant MG-63-CR-LV3-siRNA ( $1 \times 10^{7}$ cells) and SaOS-2-CR-LV3-siRNA ( $1 \times 10^{7}$ cells), respectively, at day 0 , followed by intraperitoneal injection of cisplatin $(4 \mathrm{mg} / \mathrm{kg})$ when the tumor volume reached $100 \mathrm{~mm}^{3}$. In addition, MG-63-CR-LV3 (1x10 ${ }^{7}$ cells) and SaOS-2-CR-LV3 $\left(1 \times 10^{7}\right.$ cells) were employed for in vivo experiments as control vector groups. All mice were monitored for tumor growth for 40 days before sacrifice. The tumor size in these tumor-bearing mice was measured and tumor volumes were calculated as: length $\mathrm{x}$ width ${ }^{2} \mathrm{x} 0.45$.

Histopathology assays. Tumor samples from nude mice were collected at the indicated time-points and fixed in $10 \%$ formalin solution for $48 \mathrm{~h}$, and then embedded in paraffin for the $5-\mu \mathrm{m}$-thick sections. Serial sections of the embedded specimens were stained with hematoxylin and eosin (H\&E) using standard pathology procedures and evaluated by a pathologist.

Statistical analysis. The differences between each group are expressed as the mean \pm SD. Statistical significance was assessed by Student's t-test and one-way ANOVA followed by a Tukey post hoc test. Differences were considered statistically significant at $\mathrm{P}$-value of $<0.05$.

\section{Results}

Differentially expressed LPAAT $\beta$ with strong correlation with cisplatin-resistant scenario in vivo and in vitro. Immunohistochemical detection was performed in tissue samples of both cohorts. As Fig. 1A shown, LPAAT $\beta$ in cisplatin-resistant osteosarcoma patients was stained as brown in cell membrane and nuclear while in osteosarcoma patients was very weak, similar to the control group (Fig. 1A). The cell membrane and nuclear LPAAT $\beta$ protein expression of mature and new born tumor cells was observed in the nuclear of nascent tumor cells at various differentiation states, compared to control group.

Two human osteosarcoma cell lines were subjected to treatment with 7 different concentrations of cisplatin, ranging from 0.05 to $2 \mu \mathrm{M}$ for 24,48 and $72 \mathrm{~h}$. Cytotoxicity of cisplatin in control and drug-resistant pairs (MG-63 and MG-63-CR; SaOS-2 and SaOS-2-CR) were measured by CCK8 assays. Examples of resulting volumes are shown in Fig. 1B and C. As shown in Fig. 1B, MG-63-CR cells displayed enhanced cisplatin $\mathrm{IC}_{50}$ value after cisplatin treatment, compared with those of parental cells. The $\mathrm{IC}_{50}$ value at $24 \mathrm{~h}$ was the highest compared to 48 and $72 \mathrm{~h}$. The same result is shown as Fig. 1C, SaOS-2-CR cells displayed the highest cisplatin $\mathrm{IC}_{50}$ value after cisplatin treatment for $24 \mathrm{~h}$, compared with those of parental cells. In order to extend our findings, we measured LPAAT $\beta$ level in these cell lines. LPAAT $\beta$ mRNA level and protein level were significantly increased in drug-resistant cell lines, compared with those of control parental cells, which were positively correlated with cisplatin $\mathrm{IC}_{50}$ (Fig. 1D and E). 

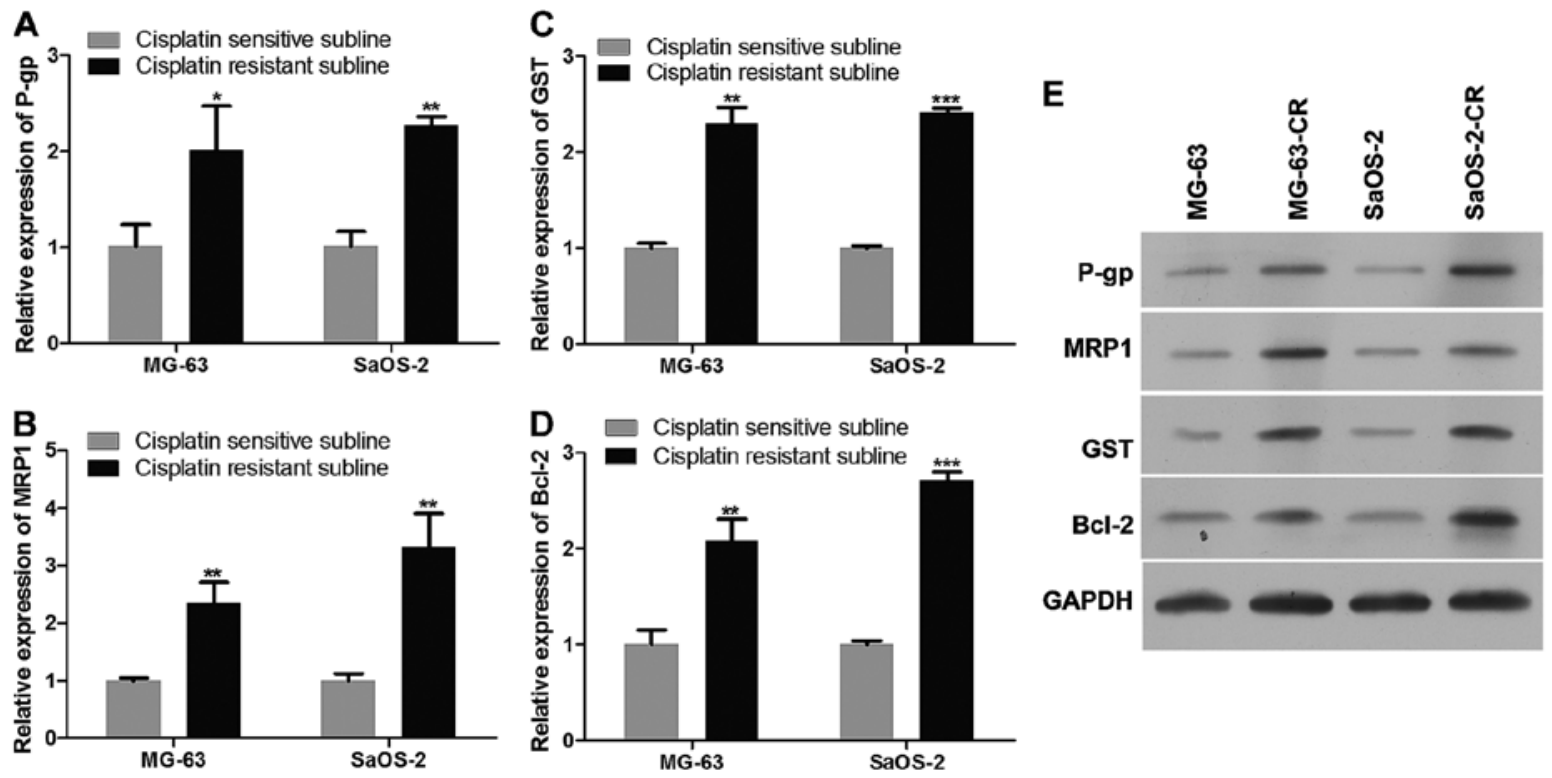

Figure 2. Expression of P-gp, MRP1, GST, Bcl-2 in both cisplatin-resistant and cisplatin-sensitive cells. (A-D) The mRNA levels of P-gp, MRP1, GST, bcl-2 in cisplatin-resistant MG-63-CR and SaOS-2-CR, as detected by real-time PCR. (E) Protein levels of P-gp, MRP1, GST, bcl-2 in cisplatin-resistant MG-63-CR and SaOS-2-CR, as detected by western blotting. All detections were repeated independently three times. ${ }^{*} \mathrm{P}<0.05,{ }^{* *} \mathrm{P}<0.01$ and ${ }^{* * * *} \mathrm{P}<0.001$ versus the parental cells ( $\mathrm{n}=3$ experiments).
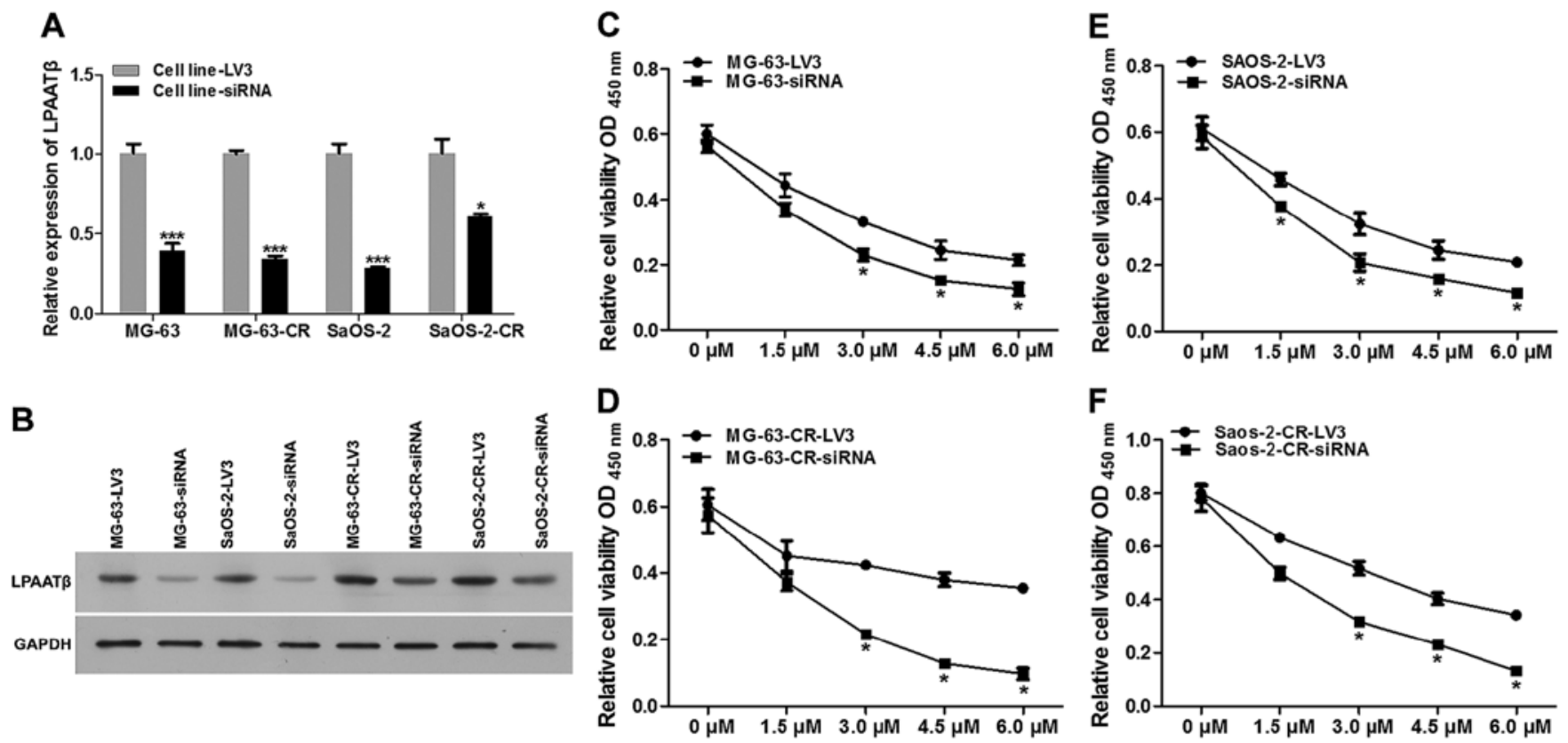

Figure 3. Effect of silencing LPAAT $\beta$ on cell viability in parental and drug-resistant cells. (A and B) Effective siRNA was screened out and validated with real-time PCR assay and western blot assay. (C and $\mathrm{D}) \mathrm{IC}_{50}$ of cisplatin in parental and drug-resistant MG-63 cells after lentivirus transfection with cisplatin treatment in different concentration. (E and F) $\mathrm{IC}_{50}$ of cisplatin in parental and drug-resistant SaOS-2 cells after lentivirus transfection with cisplatin treatment in different concentration. ${ }^{* * * *} \mathrm{P}<0.001$ vs. negative control ( $\mathrm{n}=3$ experiments).

Effect of cisplatin-resistance on expression of relevant important transporters. In order to explore the effect of cisplatin in osteosarcoma, we harvested MG-63-CR and SaOS-2-CR after cisplatin treatment for $72 \mathrm{~h}$, and then detected P-gp, MRP1, GST, Bcl-2 expression in mRNA level and protein level as Fig. 2 shows. The mRNA levels of P-gp, MRP1, GST, Bcl-2 in MG-63-CR and SaOS-2-CR were upregulated (Fig. 2A-D), compared to those of parental cells. Similar with results of
mRNA level detection, protein levels of P-gp, MRP1, GST, Bcl-2 were increased significantly (Fig. 2E).

Effect of silencing LPAAT $\beta$ on cisplatin-induced cytotoxicity in MG-63 and SaOS-2 cell lines. First we validated mRNA and protein levels of LPAAT $\beta$ after effective siRNA mediated lentivirus transfection. As shown in Fig. 3A and B, mRNA level (Fig. 3A) and protein level (Fig. 3B) were both 

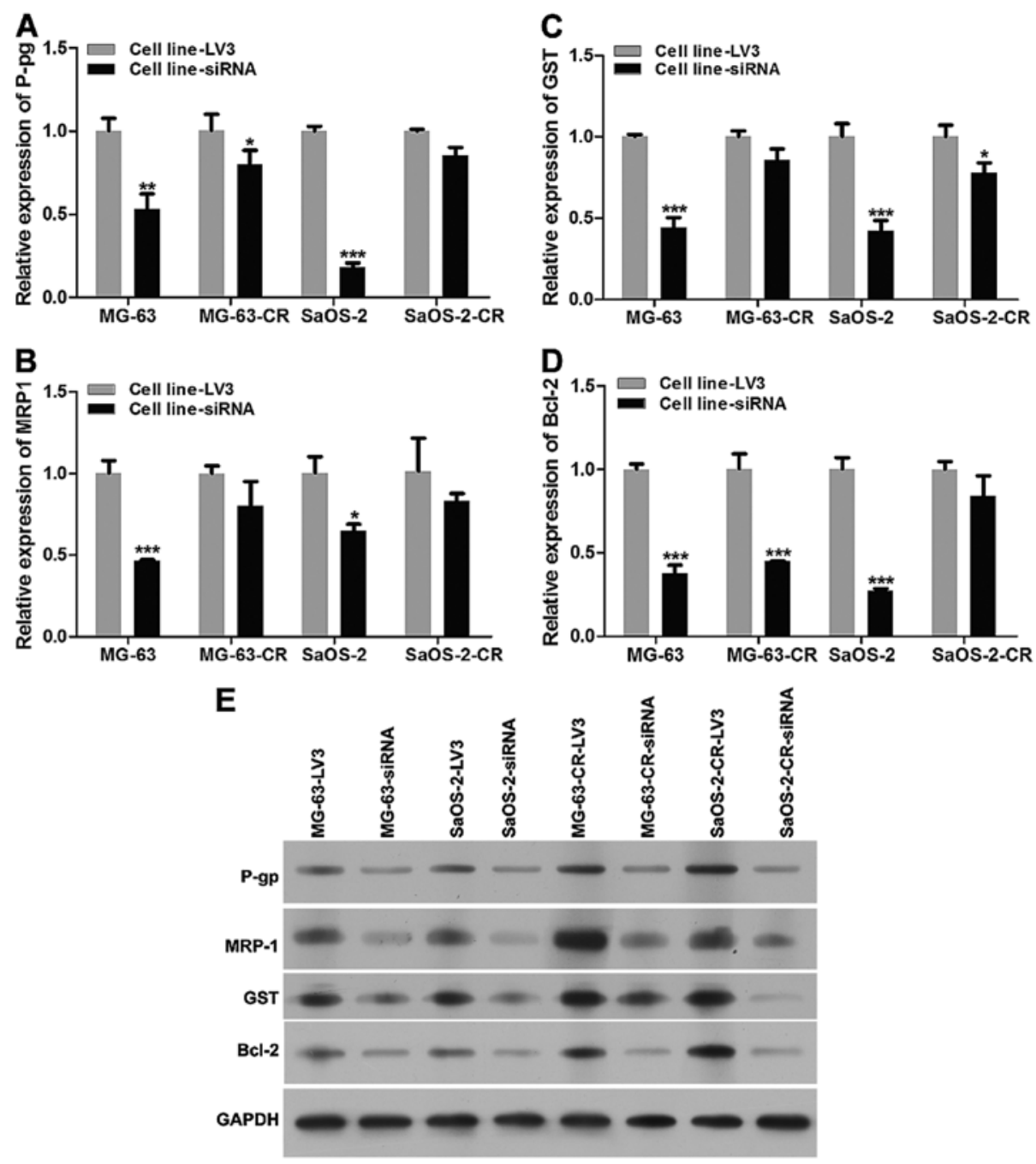

Figure 4. Effect of silencing LPAAT $\beta$ on relevant protein expression in parental and drug-resistant cells. (A) The mRNA level of P-gp in parental and drugresistant cells after lentivirus transfection. (B) The mRNA level of MRP1 in parental and drug-resistant cells after lentivirus transfection. (C) The mRNA level of GST in parental and drug-resistant cells after lentivirus transfection. (D) The mRNA level of bcl-2 in parental and drug-resistant cells after lentivirus transfection. (E) Expression of P-gp, MRP1, GST, Bcl-2 in parental MG-63 and SaOS-2, and cisplatin-resistant MG-63-CR and SaOS-2-CR, as detected by western blotting. All detections were repeated independently three times. ${ }^{* *} \mathrm{P}<0.01$ and ${ }^{* * *} \mathrm{P}<0.001$ versus the parental cells.

significantly inhibited after siRNA interference, compared to blank vector group.

To characterize the role of LPAAT $\beta$ in cisplatin resistance, two pairs of LPAAT $\beta$ knockdown cell lines from MG-63 and SaOS-2 were established, stably transfected with blank vector LV3 or effective LPAAT $\beta$ siRNA. Cytotoxicity of cisplatin in control and LPAAT $\beta$ knockdown pairs (MG-63-LV3 and MG-63-siRNA; MG-63-CR-LV3 and MG-63-CR-siRNA; SaOS-2-LV3 and SaOS-2-siRNA; SaOS-2-CR-LV3 and SaOS-2-CR-siRNA) were measured by CCK8 assays. We examined whether depletion of LPAAT $\beta$ can re-sensitize cisplatin resistant cells, as shown in Fig. 3C-F, the drug-resistant cells depleted of LPAAT $\beta$ displayed markedly reduced cisplatin $\mathrm{IC}_{50}$, compared with blank vector trasnfection groups, which was lower than those of parental cells.

Effect of silencing LPAAT及 on expression of relevant important transporters in cisplatin-resistant cell lines. We monitored the change of P-gp, MRP1, GST, Bcl-2 expression at mRNA level and protein level after LPAAT $\beta$ was silenced (Fig. 4),
mRNA levels of P-gp, MRP1, GST and Bcl-2 in parental and drug-resistant cells were downregulated (Fig. 4A-D), which is similar with result of protein level detection (Fig. 4E), compared to blank vector transfection groups.

Silencing LPAAT $\beta$ changed cellular characteristics through activating PI3K/Akt/mTOR signaling pathway. We also harvested cells after lentivirus treatment, and then detected expression of PI3K/Akt/mTOR signaling pathway relevant proteins (Fig. 5). The mRNA levels of PIK3CA, Akt1/2 and mTOR in MG-63-CR-siRNA and SaOS-2-CR-siRNA were downregulated (Fig. 5A-D), compared to those of NC group. Similar with results of mRNA level detection, protein levels of PIK3CA, p-PIK3CA, Akt1/2, p-Akt1/2, mTOR and p-mTOR were decreased significantly (Fig. 5E).

Immunofluorescence results showed that phospho-mTOR expression at $48 \mathrm{~h}$ was increased significantly in MG-63-CR cells (Fig. 6A) and SaOS-2-CR cells (Fig. 6B) compared to their parental cell lines, which were downregulated in MG-63-CR cells (Fig. 6A) and SaOS-2-CR cells (Fig. 6B) 

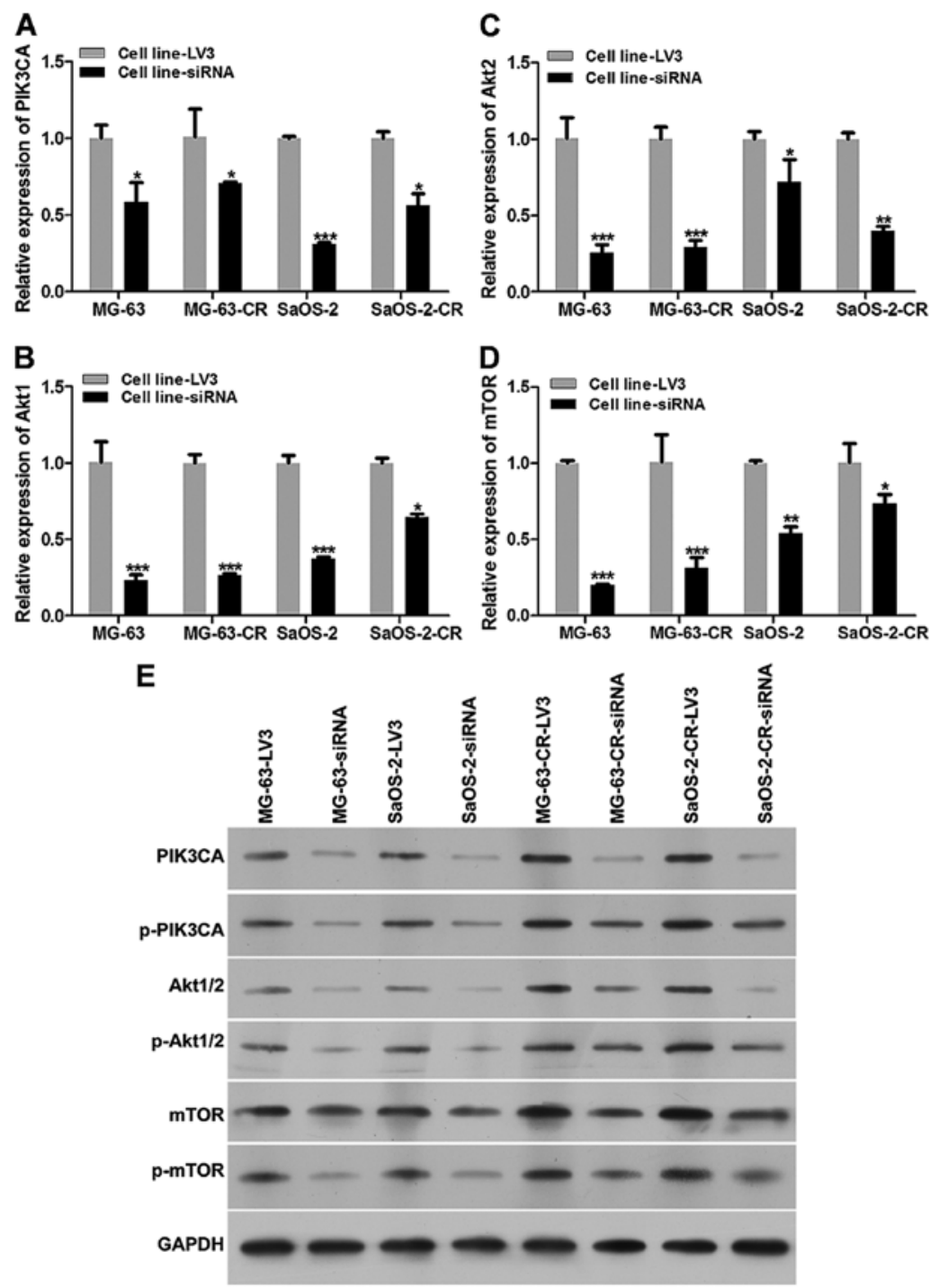

Figure 5. Effect of silencing LPAAT $\beta$ on PI3K/Akt/mTOR signaling pathway in parental and drug-resistant cells. (A) The mRNA level of P-gp in parental and drug-resistant cells after lentivirus transfection. (B) The mRNA level of MRP1 in parental and drug-resistant cells after lentivirus transfection. (C) The mRNA level of GST in parental and drug-resistant cells after lentivirus transfection. (D) The mRNA level of bcl-2 in parental and drug-resistant cells after lentivirus transfection. (E) Expression of PIK3CA, p-PIK3CA, Akt1/2, p-Akt1/2, mTOR, p-mTOR in parental MG-63 and SaOS-2, and cisplatin-resistant MG-63-CR and SaOS-2-CR after lentivirus transfection, as detected by western blotting. ${ }^{*} \mathrm{P}<0.05,{ }^{* *} \mathrm{P}<0.01$ and ${ }^{* * *} \mathrm{P}<0.001$ versus the parental cells ( $\mathrm{n}=3$ experiments).

transfected with siRNA mediated by lentivirus compared with negative control.

In vivo LPAAT $\beta$ gene silencing using lentivirus as a potential strategy for cisplatin resistance. Using a nude mouse model, it was found that mice with subcutaneous LPAAT $\beta$-depleted cell line derived tumors had smaller tumor burden, with consequent enhanced survival benefit (Fig. 7A and B). Interestingly, we monitored the tumor volumn after cells implantation for 40 days and found that tumor volume in MG-63/SaOS-2siRNA group was significantly inhibited with respect of MG-63-LV3/SaOS-2-LV3 group, based on pair comparison at every time-point (Fig. 7C). Moreover, extensive tubular necrosis was observed in the nude mice with implantation of drug re-sensitive cancer cells by lentivirus-mediated siRNA insertion using histopathological examinations (Fig. 7D). Immunohistochemical detection displayed phospho-mTOR expression alteration in both cisplatin-resistant cells and cisplatin re-sensitive cells. As Fig. 7E shows, phospho-mTOR in cisplatin-resistant osteosarcoma cells (MG-63-CR-LV3 group and SaOS-2-CR-LV3 group) was stained brown in cell membrane and nuclear while in cisplatin re-sensitive cells was very weak (MG-63-CR-siRNA group and SaOS-2-CR-RNA group).

\section{Discussion}

As the mostcommon primarymalignancy of bone,osteosarcoma has complicated occurrence and development which have not been clarified yet. Although development of surgery combined with neoadjuvant chemotherapy has significantly improved, the survival rate of osteosarcoma in the last few decades has plateaued, which may be heavily influenced by resistance to chemotherapy drugs $(16,17)$. Therefore, discovery of effective ways that can increase the sensitivity to chemotherapy will be important in antitumor effect. In this study, we investigated 

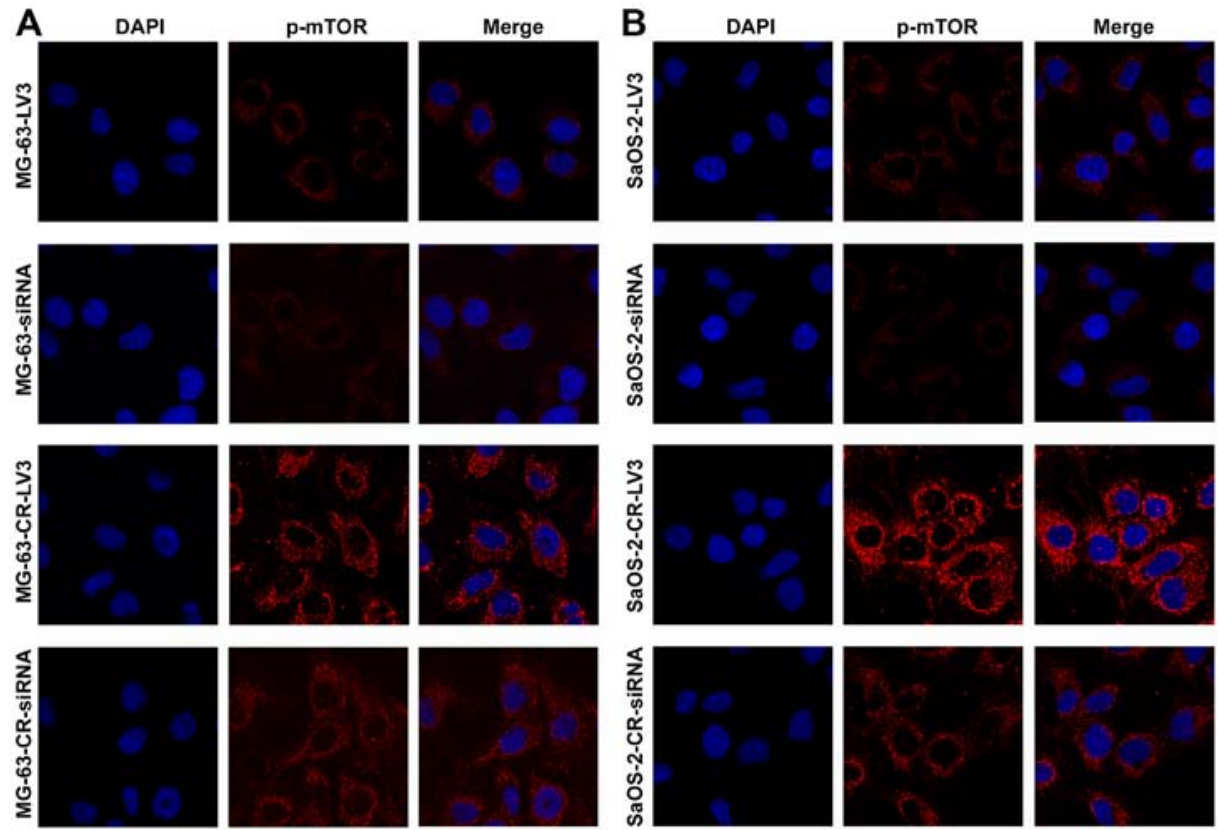

Figure 6. Expression of phospho-mTOR. (A and B) Expression of phospho-mTOR in both cisplatin-resistant cells (MG-63-CR/MG-63-CR-LV3 and SaOS-2-CR/SaOS-2-CR-LV3) and cisplatin re-sensitive cells (MG-63-CR-siRNA and SaOS-2-CR-siRNA) at $48 \mathrm{~h}$ after transfection with lentivirus inserted with LPAAT $\beta$ siRNA and the blank vector LV3 as negative control by immunofluorescence staining, $\mathrm{x} 400$ magnification.
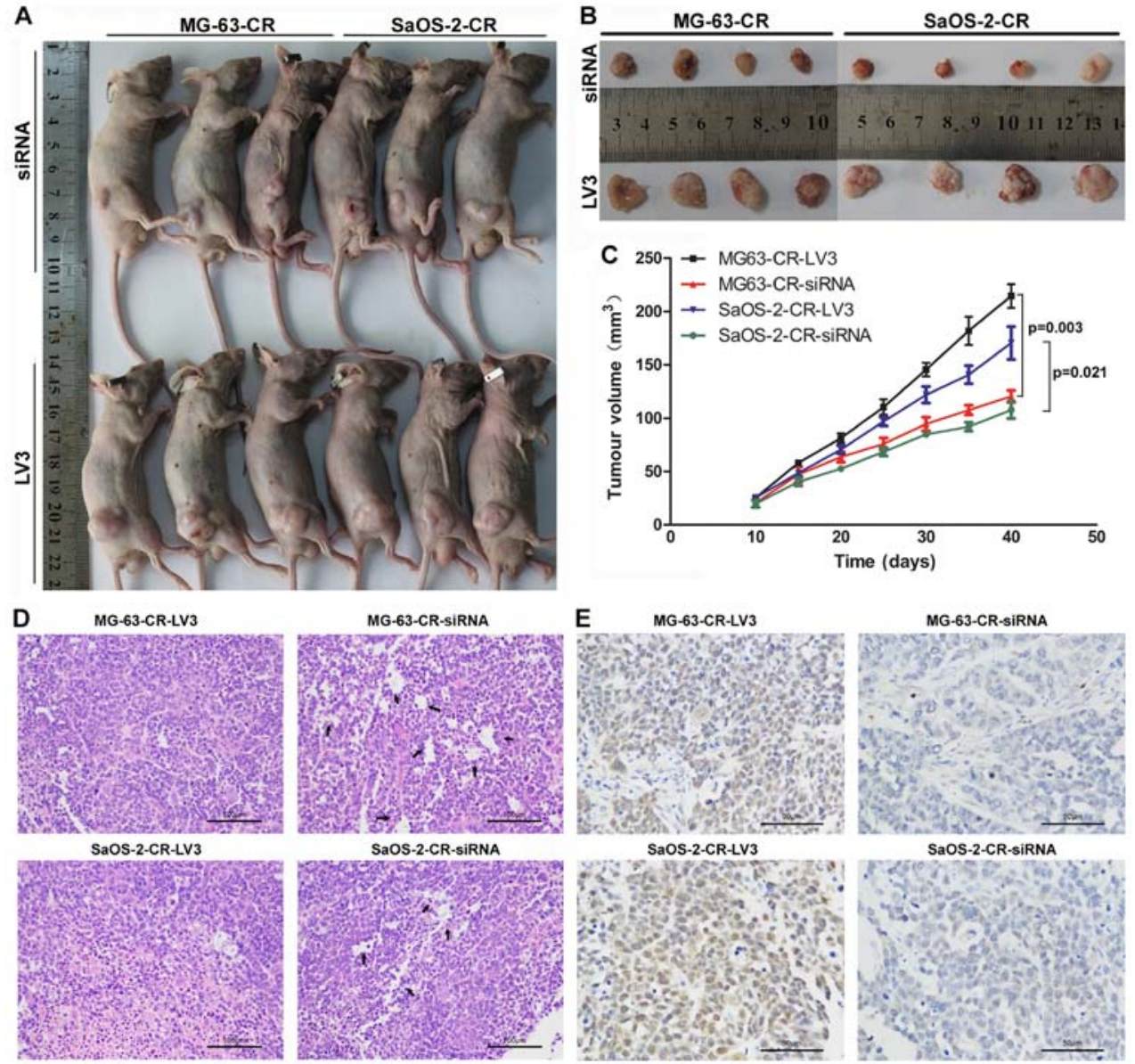

Figure 7. Tumor growth in vivo. (A) Images of tumor burden in mice implanted with lentivitus-infected MG-63-CR and SaOS-2-CR cells for 40 days and treated in vivo using intratumoral injection of cisplatin at a dose of $4 \mathrm{mg} / \mathrm{kg}$ when tumor volumn reached $100 \mathrm{~mm}^{3}$ during treatment. (B) Presentation of tumor with partial enlargement. MG-63-CR and SaOS-2-CR cells transfected with blank scrambled sequence siRNA were considered as negative control (C) Perpetual records of tumor volumn in all groups of nude mice were taken from day 10 to day 40 after implantation accomplished. (D) Histological analysis of all nude mice with different implantation by H\&E staining. Arrowheads, tubular necrosis. Scale bar represents $100 \mu \mathrm{m}$. (E) The nucleus and membrane of small cells and mature tumor cells are stained brown with phospho-mTOR as conducted by immunohistochemistry assay. Scale bar represents $50 \mu \mathrm{m}$. 
the role of LPAAT $\beta$ in osteosarcoma with cisplatin-resistance scenario. The results show that LPAAT $\beta$ had high expression in osteosarcoma patients who received cisplatin treatment and cisplatin-resistant MG-63-CR/SaOS-2-CR cells and the $\mathrm{IC}_{50}$ was higher than in the control groups. Expression of some important relevant proteins including P-gp, MRP1, GST, Bcl-2 were downregulated after downregulating LPAAT $\beta$ expression mediated by lentivirus. Also silencing LPAAT $\beta$ triggered the PI3K/Akt/mTOR signaling pathway in cisplatin-resistant MG-63-CR/SaOS-2-CR cells with lower $\mathrm{IC}_{50}$ compared to control group. Using the nude mouse model bearing osteosarcoma tumor xenografts of cisplantin-resistant osteosarcoma cells, we demonstrated that silencing LPAAT $\beta$ effectively inhibited osteosarcoma tumor growth. These results suggested that LPAAT $\beta$ may play an important role of lowering the sensitivity to chemotherapy in regulating cisplatin-resistant osteosarcoma cell proliferation through activating PI3K/Akt/ mTOR signaling pathway. Thus, our results confirm the role of LPAAT $\beta$ in osteosarcoma growth.

It has been shown that inhibition of LPAAT $\beta$ may play an important role in regulating osteosarcoma cell proliferation and tumor growth through catalysing antitumor activity, which usually results in an arrest of cell signaling pathways and apoptosis $(15,18)$. Previous studies have indicated that inhibition of LPAAT $\beta$ expression via siRNA interfering suppresses basal Erk phosphorylation, prevents the translocation of Raf to the plasma Erk phosphorylation and inhibits the activation of proteins in the phosphoinositide-3-kinase/ Akt pathway, including Akt, mTOR, and S6 kinase $(12,19)$. Therefore, LPAAT $\beta$ may be considered and exploited as a novel therapeutic target for osteosarcoma clinical management, as indicated consistently in this study.

Previous research revealed that aberrant activation of PI3K/ Akt/mTOR signaling pathway usually plays a pivotal role in malignant transformation and chemoresistance for cancer cells through regulating multidrug resistance gene $1 / \mathrm{P}$-glycoprotein (MDR1/P-gp) (20-22). PI3K stimulated by multiple growth factors contributes to chronic activation of Akt in cancer cells, downstream of which both activate the mTOR kinase $(23,24)$. It was reported that VEGF expression in different tumors for angiogenesis can be regulated by activating PI3K/Akt/ mTOR signal pathway (25). Through negative regulation of P53, the activated Akt protein induces cisplatin resistance in ovarian cancer, which could be reversed by PI3K inhibitor to increase the mitochondrial Bax translocation and cytC release $(26,27)$. Other studies also demonstrated that higher mTOR phosphorylation is involved in cisplatin resistance with strong sensitivity to mTOR inhibitor in vitro, which was validated in our results (28). Also the activation of the PI3K/Akt/mTOR signaling pathway was indicated to inhibit cisplatin-induced apoptosis and improve cisplatin resistance in cancer cells (28-30).

Our results in vitro indicate that LPAAT $\beta$ may increase cisplatin-resistant osteosarcoma cell proliferation and viability which may be reversed by silencing LPAAT $\beta$, with significant variation of relevant proteins and activation of PI3K/Akt/ mTOR signally pathway. Therefore, we chose a nude mouse model to further investigate the effect of LPAAT $\beta$ on tumor growth and found silencing LPAAT $\beta$ significantly inhibited the osteosarcoma tumor growth in vivo after cisplatin-resistant osteosarcoma cell transplantation. Subsequently, targeting LPAAT $\beta$ may be exploited as a novel therapeutic strategy for osteosarcoma clinical treatment, which is especially attractive given the availability of selective pharmacological inhibitors.

\section{Acknowledgements}

This study was supported by grants from the National Natural Science Foundation of China (no. 81302347) and the National Natural Science Foundation of China (no. 81400418).

\section{References}

1. Alcantara MB, Nemazannikova N, Elahy M and Dass CR: Pigment epithelium-derived factor upregulates collagen I and downregulates matrix metalloproteinase 2 in osteosarcoma cells, and colocalises to collagen I and heat shock protein 47 in fetal and adult bone. J Pharm Pharmacol 66: 1586-1592, 2014.

2. Fortunati D, Reppe S, Fjeldheim AK, Nielsen M, Gautvik VT and Gautvik KM: Periostin is a collagen associated bone matrix protein regulated by parathyroid hormone. Matrix Biol 29: 594-601, 2010

3. Ferrari D, Codecà C, Battisti N, Broggio F, Crepaldi F, Violati M, Bertuzzi C, Dottorini L, Caldiera S, Luciani A, et al: Multimodality treatment of osteosarcoma of the jaw: A single institution experience. Med Oncol 31: 171, 2014.

4. Yu W, Tang L, Lin F, Li D, Wang J, Yang Y and Shen Z: Stereotactic radiosurgery, a potential alternative treatment for pulmonary metastases from osteosarcoma. Int J Oncol 44: 1091-1098, 2014.

5. Li Z, Zhao L and Wang Q: Overexpression of long non-coding RNA HOTTIP increases chemoresistance of osteosarcoma cell by activating the Wnt/ $\beta$-catenin pathway. Am J Transl Res 8: 2385-2393, 2016.

6. Yu L, Fan Z, Fang S, Yang J, Gao T, Simões BM, Eyre R, Guo W and Clarke RB: Cisplatin selects for stem-like cells in osteosarcoma by activating Notch signaling. Oncotarget 7: 33055-33068, 2016.

7. Dell'Amore A, Asadi N, Caroli G, Dolci G, Bini A and Stella F: Recurrent primary cardiac osteosarcoma: A case report and literature review. Gen Thorac Cardiovasc Surg 62: 175-180, 2014.

8. Hawkins DS and Arndt CA: Pattern of disease recurrence and prognostic factors in patients with osteosarcoma treated with contemporary chemotherapy. Cancer 98: 2447-2456, 2003.

9. Pagel JM, Laugen C, Bonham L, Hackman RC, Hockenbery DM, Bhatt R, Hollenback D, Carew H, Singer JW and Press OW: Induction of apoptosis using inhibitors of lysophosphatidic acid acyltransferase-beta and anti-CD20 monoclonal antibodies for treatment of human non-Hodgkin's lymphomas. Clin Cancer Res 11: 4857-4866, 2005.

10. Bonham L, Leung DW, White T, Hollenback D, Klein P Tulinsky J, Coon M, de Vries P and Singer JW: Lysophosphatidic acid acyltransferase-beta: A novel target for induction of tumour cell apoptosis. Expert Opin Ther Targets 7: 643-661, 2003.

11. Schmid B, Finnen MJ, Harwood JL and Jackson SK: Acylation of lysophosphatidylcholine plays a key role in the response of monocytes to lipopolysaccharide. Eur J Biochem 270: 2782-2788, 2003.

12. Blaskovich MA, Yendluri V, Lawrence HR, Lawrence NJ, Sebti SM and Springett GM: Lysophosphatidic acid acyltransferase beta regulates mTOR signaling. PLoS One 8: e78632, 2013.

13. Douvas MG, Hogan KN, Ji Y, Hollenback D, Bonham L, Singer JW and Mitchell BS: Effect of lysophosphatidic acid acyltransferase-beta inhibition in acute leukemia. Leuk Res 30: 1027-1036, 2006.

14. Song L, Yang J, Duan P, Xu J, Luo X, Luo F, Zhang Z, Hou T, Liu B and Zhou Q: MicroRNA-24 inhibits osteosarcoma cell proliferation both in vitro and in vivo by targeting LPAAT $\beta$. Arch Biochem Biophys 535: 128-135, 2013.

15. Rastegar F, Gao JL, Shenaq D, Luo Q, Shi Q, Kim SH, Jiang W, Wagner ER, Huang E, Gao Y, et al: Lysophosphatidic acid acyltransferase $\beta$ (LPAAT $\beta$ ) promotes the tumor growth of human osteosarcoma. PLoS One 5: e14182, 2010. 
16. Huang Z, Huang $\mathrm{Y}, \mathrm{He} \mathrm{H}$ and $\mathrm{Ni}$ J: Podocalyxin promotes cisplatin chemoresistance in osteosarcoma cells through phosphatidylinositide 3-kinase signaling. Mol Med Rep 12: 3916-3922, 2015

17. Ma Y, Ren Y, Han EQ, Li H, Chen D, Jacobs JJ, Gitelis S, O'Keefe RJ, Konttinen YT, Yin G, et al: Inhibition of the Wnt- $\beta$ catenin and Notch signaling pathways sensitizes osteosarcoma cells to chemotherapy. Biochem Biophys Res Commun 431: 274-279, 2013.

18. Körbes AP, Kulcheski FR, Margis R, Margis-Pinheiro M and Turchetto-Zolet AC: Molecular evolution of the lysophosphatidic acid acyltransferase (LPAAT) gene family. Mol Phylogenet Evol 96: 55-69, 2016

19. Coon M, Ball A, Pound J, Ap S, Hollenback D, White T, Tulinsky J, Bonham L, Morrison DK, Finney R, et al: Inhibition of lysophosphatidic acid acyltransferase beta disrupts proliferative and survival signals in normal cells and induces apoptosis of tumor cells. Mol Cancer Ther 2: 1067-1078, 2003.

20. Ma Q, Chang Z, Wang W and Wang B: Rapamycin-mediated mTOR inhibition reverses drug resistance to adriamycin in colon cancer cells. Hepatogastroenterology 62: 880-886, 2015.

21. Marklein D, Graab U, Naumann I, Yan T, Ridzewski R, Nitzki F, Rosenberger A, Dittmann K, Wienands J, Wojnowski L, et al: PI3K inhibition enhances doxorubicin-induced apoptosis in sarcoma cells. PLoS One 7: e52898, 2012.

22. Randle RA, Raguz S, Higgins CF and Yagüe E: Role of the highly structured 5'-end region of MDR1 mRNA in P-glycoprotein expression. Biochem J 406: 445-455, 2007.

23. Wang SF, Chou YC, Mazumder N, Kao FJ, Nagy LD, Guengerich FP, Huang C, Lee HC, Lai PS and Ueng YF: 7-Ketocholesterol induces P-glycoprotein through PI3K/mTOR signaling in hepatoma cells. Biochem Pharmacol 86: 548-560, 2013.
24. Markova B, Albers C, Breitenbuecher F, Melo JV, Brümmendorf TH, Heidel F, Lipka D, Duyster J, Huber C and Fischer T: Novel pathway in Bcr-Abl signal transduction involves Akt-independent, PLC-gamma1-driven activation of mTOR/ p70S6-kinase pathway. Oncogene 29: 739-751, 2010

25. Tsai JL, Lee YM, Pan CY and Lee AY: The novel VEGF121VEGF165 fusion attenuates angiogenesis and drug resistance via targeting VEGFR2-HIF-1 $\alpha$-VEGF165/Lon signaling through PI3K-AKT-mTOR pathway. Curr Cancer Drug Targets 16: 275-286, 2016.

26. Zhao Z, Wang J, Tang J, Liu X, Zhong Q, Wang F, Hu W, Yuan Z, Nie C and Wei Y: JNK- and Akt-mediated Puma expression in the apoptosis of cisplatin-resistant ovarian cancer cells. Biochem J 444: 291-301, 2012.

27. Fraser M, Leung BM, Yan X, Dan HC, Cheng JQ and Tsang BK: p53 is a determinant of X-linked inhibitor of apoptosis protein/ Akt-mediated chemoresistance in human ovarian cancer cells. Cancer Res 63: 7081-7088, 2003.

28. Yuge K, Kikuchi E, Hagiwara M, Yasumizu Y, Tanaka N, Kosaka T, Miyajima A and Oya M: Nicotine induces tumor growth and chemoresistance through activation of the PI3K/ Akt/mTOR pathway in bladder cancer. Mol Cancer Ther 14: 2112-2120, 2015.

29. Gan Y, Wang Y, Tan Z, Zhou J, Kitazawa R, Jiang X, Tang Y and Yang J: TDRG1 regulates chemosensitivity of seminoma TCam-2 cells to cisplatin via PI3K/Akt/mTOR signaling pathway and mitochondria-mediated apoptotic pathway. Cancer Biol Ther 17: 741-750, 2016.

30. Cai Y, Tan X, Liu J, Shen Y, Wu D, Ren M, Huang P and Yu D: Inhibition of PI3K/Akt/mTOR signaling pathway enhances the sensitivity of the SKOV3/DDP ovarian cancer cell line to cisplatin in vitro. Chin J Cancer Res 26: 564-572, 2014. 